\title{
THE DEFORMATION THEORY OF REPRESENTATIONS OF FUNDAMENTAL GROUPS OF COMPACT KÄHLER MANIFOLDS
}

\author{
WILLIAM M. GOLDMAN AND JOHN J. MILLSON
}

We work over a fixed field $\mathbf{k}$, either the real or complex numbers. A quadratic cone in a $\mathbf{k}$-vector space $E$ is an affine variety $\mathcal{Q} \subset E$ defined by equations

$$
B(u, u)=0
$$

where $B: E \times E \rightarrow E^{\prime}$ is a bilinear map and $E^{\prime}$ is a vector space. ( $E$ may be identified with the Zariski tangent space to $\mathcal{Q}$ at 0 .) Let $V$ be an algebraic variety and $x \in V$ be a point. We say that $V$ is quadratic at $x$ if the analytic germ of $V$ at $x$ is equivalent to the germ of a quadratic cone at 0 .

Let $\Gamma$ be a finitely generated group and $G$ a k-algebraic group. We identify $G$ with its set of k-points, which has the natural structure of a Lie group over k. Then the set $\operatorname{Hom}(\Gamma, G)$ of all homomorphisms $\Gamma \rightarrow G$ equals the set of k-points of a k-algebraic variety $\mathfrak{R}(\Gamma, G)$ (compare $[\mathbf{9}, 10]$ ).

THEOREM 1. Let $\Gamma$ be the fundamental group of a compact Kähler manifold. Let $\rho \in \operatorname{Hom}(\Gamma, G)$ be a representation such that its image $\rho(\Gamma)$ lies in a compact subgroup of $G$. Then $\mathfrak{R}(\Gamma, G)$ is quadratic at $\rho$.

Suppose that $\left\{H^{p, q}, Q\right\}$ is a polarized Hodge structure of weight $n$, that $G$ is the group of real points of the isometry group of $Q$ and $X=G / V$ is the classifying space for polarized Hodge structures of the above type (see Griffiths [8, p. 15]). Suppose further $M$ is a complex manifold with fundamental group $\Gamma$. A representation $\rho: \Gamma \rightarrow G$ determines a flat principal $G$-bundle $P_{\rho}$ over $M$ and an associated $X$-bundle $P_{\rho} \times_{G} X$. A horizontal holomorphic $V$ reduction of $P_{\rho}$ is a holomorphic section of $P_{\rho} \times_{G} X$ whose differential carries the holomorphic tangent bundle of $M$ into the horizontal subbundle $T_{h}(X)$ defined in $[8$, p. 20].

THEOREM 2. Let $M$ be a compact Kähler manifold with fundamental group $\Gamma$ and $X=G / V$ a classifying space for polarized Hodge structures. Suppose that $\rho: \Gamma \rightarrow G$ is a representation such that the associated principal bundle over $M$ admits a horizontal holomorphic $V$-reduction. Then $\mathfrak{R}(\Gamma, G)$ is quadratic at $\rho$.

Received by the editors October 15, 1987.

1980 Mathematics Subject Classification (1985 Revision). Primary 53C55, 57M05; Secondary $14 \mathrm{C} 30$, 32J25.

The first author was supported in part by National Science Foundation grant DMS86-13576 and an Alfred P. Sloan Foundation Fellowship; the second by National Science Foundation grant DMS-85-01742. 
If $\pi: E \rightarrow M$ is a holomorphic family of smooth polarized projective varieties parametrized by $M$, then the period mapping which attaches to $x \in M$ the polarized Hodge structure on $H^{n}\left(\pi^{-1}(x)\right)$ is a horizontal holomorphic $V$-reduction of the principal bundle associated to the monodromy representation.

COROLlaRY. Suppose $\rho: \Gamma \rightarrow G$ is the monodromy of a variation of Hodge structure over $M$. Then $\mathfrak{R}(\Gamma, G)$ is quadratic at $\rho$.

The idea behind the proof of Theorem 2 can be applied to a number of other closely related situations. The following result can be used to construct deformations of discrete groups of automorphisms of complex hyperbolic space (compare [2] and [6]).

THEOREM 3. Let $M$ be a compact Kähler manifold with fundamental group $\Gamma$ and $X=G / K$ a Hermitian symmetric space with automorphism group $G$. Suppose $\rho: \Gamma \rightarrow G$ is a representation such that the associated principal $G$ bundle over $M$ admits a holomorphic K-reduction. Then $\mathfrak{R}(\Gamma, G)$ is quadratic at $\rho$.

Perhaps the most important feature of such "quadratic singularity" theorems is that the quadratic functions are computable algebraic topological invariants. Thus we obtain a criterion for nonsingularity of $\mathfrak{R}(\Gamma, G)$ near a representation $\rho$. The Zariski tangent space to $\mathfrak{R}(\Gamma, G)$ near $\rho$ equals the space $Z^{1}\left(\Gamma ; \mathfrak{g}_{\text {Ad } \rho}\right)$ of Eilenberg-Mac Lane 1-cocycles of $\Gamma$ with coefficients in the $\Gamma$ module $\mathfrak{g}_{\text {Ad } \rho}$ (given the action defined by the composition $\Gamma \rightarrow G \rightarrow \operatorname{Aut}(\mathfrak{g})$ ). The quadratic cone is defined by the cup-product where the Lie bracket $[]:, \mathfrak{g} \times \mathfrak{g} \rightarrow \mathfrak{g}$ is used as a coefficient pairing.

COROLLARY. Suppose that $M$ is a compact Kähler manifold with fundamental group $\Gamma$ and that $\rho: \Gamma \rightarrow G$ is a representation satisfying the hypotheses of Theorems 1,2, or 3. Suppose that the cup-product

$$
H^{1}\left(\Gamma, \mathfrak{g}_{\text {Ad } \rho}\right) \times H^{1}\left(\Gamma, \mathfrak{g}_{\text {Ad } \rho}\right) \rightarrow H^{2}\left(\Gamma, \mathfrak{g}_{\text {Ad } \rho}\right)
$$

is identically zero. Then $\mathfrak{R}(\Gamma, G)$ is nonsingular at $\rho$.

For $G$ compact, the quadraticity of tangent cones to $\Re(\Gamma, G)$ was proved in Goldman-Millson [7]. When $\rho$ is a reductive representation of the fundamental group of a closed surface, it was shown in Goldman [5] that the tangent cone to $\mathfrak{R}(\Gamma, G)$ is quadratic. Recent work of Carlos Simpson (Higgs bundles and local systems. II, Princeton Univ. preprint) implies quadratic singularities for a large class of representations, including reductive representations of surface groups.

The proofs of Theorems 1, 2, and 3 involve showing that two analytic germs are equivalent. We replace an analytic germ $(V, x)$ first by the complete local k-algebra $\hat{O}_{(V, x)}$ of $V$ at $x$; it follows from Artin [1] that the isomorphism class of $\hat{O}_{(V, x)}$ determines the equivalence class of the analytic germ of $V$ at $x$. Furthermore this isomorphism class is determined by the functor which associates to an Artin local k-algebra $A$ the set $\operatorname{Hom}\left(\hat{O}_{(V, x)}, A\right)$ of k-algebra homomorphisms $\operatorname{Hom}\left(\hat{O}_{(V, x)}, A\right)$, or equivalently the set of $A$-points of $V$ over 
$x \in V$. Thus we prove two analytic germs are equivalent by showing their corresponding functors $\mathbf{A}_{\mathbf{k}}^{\text {local }} \rightarrow$ Sets are naturally isomorphic where $\mathbf{A}_{\mathbf{k}}^{\text {local }}$ denotes the category of Artin local $\mathbf{k}$-algebras.

This will be accomplished by introducing several auxiliary "deformation theories." A transformation groupoid is a category $C$ arising from the action of a group on the set Obj $C$ of objects of $C$. If $C$ arises from an action of a group $H$ and $Y$ is another $H$-set then we denote by $C \bowtie Y$ the transformation groupoid arising from the diagonal action of $H$ on $\mathrm{Obj} C \times Y$. If $F: C \rightarrow C^{\prime}$ is a functor of transformation groupoids preserving actions of the corresponding groups on a set $Y$, then there is a corresponding functor $F \bowtie Y: C \bowtie Y \rightarrow C^{\prime} \bowtie Y$; then $F \bowtie Y$ is an equivalence of categories if $F$ is. If $C$ is a category we denote by Iso $C$ the collection of isomorphism classes of objects; an equivalence of groupoids $C \rightarrow C^{\prime}$ induces an isomorphism of sets Iso $C \rightarrow$ Iso $C^{\prime}$.

Fix an Artin local k-algebra $A$ with maximal ideal $\mathfrak{m}$; let $G_{A}$ denote the group of $A$-points of $G$ with its natural structure as a Lie group over $\mathbf{k}$-indeed $G_{A}$ equals the semidirect product of $G$ with the nilpotent normal subgroup $\exp (\mathfrak{g} \otimes \mathfrak{m})$. If $\rho_{0} \in \operatorname{Hom}(\Gamma, G)$ then the set of $A$-points of $\mathfrak{R}(\Gamma, G)$ over $\rho_{0}$ equals the fiber $q_{*}^{-1}\left(\rho_{0}\right)$ of the map

$$
q_{*}: \operatorname{Hom}\left(\Gamma, G_{A}\right) \rightarrow \operatorname{Hom}(\Gamma, G)
$$

induced by projection $q: G_{A} \rightarrow G$. Let $R_{A}\left(\rho_{0}\right)$ (resp. $R_{A}^{\prime}\left(\rho_{0}\right)$ ) denote the transformation groupoid with set of objects $q_{*}^{-1}\left(\rho_{0}\right)$, and with morphisms conjugation by elements of $\exp (\mathfrak{g} \otimes \mathfrak{m})$ (resp. only the identity morphisms).

Let $M$ be a manifold, $x \in M$ and let $\Gamma=\pi_{1}(M, x)$. Let $\left(P, \omega_{0}\right)$ be the flat principal $G$-bundle over $M$ with holonomy representation $\rho_{0}$. Let $P_{A}=P \times_{G}$ $G_{A}$ be the principal $G_{A}$-bundle obtained by enlarging the structure group of $P$ by $G \rightarrow G_{A}$ and let $i: P \rightarrow P_{A}$ be the corresponding inclusion; then the flat connection $\omega_{0}$ on $P$ induces a flat connection $\tilde{\omega}_{0}$ on $P_{A}$. Let $\mathcal{F}_{A}\left(\omega_{0}\right)$ (resp. $\mathcal{F}_{A}^{\prime}\left(\omega_{0}\right)$ ) denote the transformation groupoid with objects the flat connections $\tilde{\omega}$ on $P_{A}$ such that $i^{*} \tilde{\omega}=\omega_{0}$ and morphisms the gauge transformations $P_{A} \rightarrow$ $P_{A}$ which act trivially on $P \subset P_{A}$ (resp. and fix the fiber over $x$ ). Choose a basepoint $p$ in the fiber of $P_{A}$ over $x$. If $F$ is a gauge transformation (resp. infinitesimal gauge transformation) of $P_{A}$, let $\varepsilon_{p}(F)$ denote the element of $G$ (resp. $\mathfrak{g}$ ) corresponding to the action of $F$ at $p$. If $\omega$ is a flat connection on $P_{A}$, let $\operatorname{hol}_{p}(\omega) \in \operatorname{Hom}\left(\Gamma, G_{A}\right)$ denote the holonomy homomorphism of $\omega$ at $p$.

LEMMA. The correspondence $\left(\operatorname{hol}_{p}, \varepsilon_{p}\right)$ defines equivalences of categories

$$
\digamma_{A}\left(\omega_{0}\right) \rightarrow R_{A}\left(\rho_{0}\right)
$$

and

$$
F_{A}^{\prime}\left(\omega_{0}\right) \rightarrow R_{A}^{\prime}\left(\rho_{0}\right)
$$

which depend naturally on $A$.

Thus we replace the functor $A \mapsto$ Iso $R_{A}^{\prime}\left(\rho_{0}\right)$ corresponding to the germ of $\Re(\Gamma, G)$ at $\rho_{0}$ by the naturally isomorphic functor $A \mapsto$ Iso $\mathcal{F}_{A}^{\prime}\left(\omega_{0}\right)$. We next reinterpret the functor $A \mapsto \mathcal{F}_{A}^{\prime}\left(\omega_{0}\right)$ in the general context of differential graded Lie algebras. 
Let $L$ denote a differential graded Lie algebra; following Deligne [3] we define a functor $A \mapsto C(L ; A)$ from $\mathbf{A}_{\mathbf{k}}^{\text {local }}$ to the category of transformation groupoids as follows. The objects of $C(L ; A)$ are the elements $\eta \in L^{1} \otimes \mathfrak{m}$ satisfying the deformation equation

$$
d \eta+\frac{1}{2}[\eta, \eta]=0
$$

and the morphisms are elements of the nilpotent group $\exp \left(L^{0} \otimes \mathfrak{m}\right)$ acting affinely on $L^{1} \otimes \mathfrak{m}$ by

$$
\exp (t \lambda): \eta \mapsto \exp (t \operatorname{ad} \lambda)(\eta)+\frac{I-\exp (t \operatorname{ad} \lambda)}{\operatorname{ad} \lambda}(d \lambda)
$$

If $\mathfrak{g}$ is a Lie algebra and $L$ is a differential graded Lie algebra, then a $\mathfrak{g}$ augmentation of $L$ is a Lie algebra homomorphism $\varepsilon: L \rightarrow \mathfrak{g}$, where $\mathfrak{g}$ is regarded as a differential graded Lie algebra with no nonzero elements of positive degree. The augmentation ideal is $L^{\prime}=\operatorname{Ker} \varepsilon$. We obtain an equivalence of categories

$$
C\left(L^{\prime} ; A\right) \rightarrow C(L ; A) \bowtie \exp (\mathfrak{g} \otimes \mathfrak{m})
$$

depending naturally on $A$.

Two $g$-augmented differential graded Lie algebras are quasi-isomorphic if they can be connected by a sequence of homomorphisms of $\mathfrak{g}$-augmented differential graded Lie algebras each inducing an isomorphism on cohomology; a g-augmented differential graded Lie algebra is formal if it is quasi-isomorphic to one with zero differential. The following basic result states that quasiisomorphic differential graded Lie algebras give rise to equivalent deformation theories:

TheOREM (SChlessinger-STAShefF [12], Deligne [3]). Let $\mathbf{k}$ be a field of characteristic zero and $\varphi: L \rightarrow \bar{L}$ be a homomorphism of differential graded Lie algebras such that the induced maps $H^{i}(\varphi): H^{i}(L) \rightarrow H^{i}(\bar{L})$ are isomorphisms for $i=0,1$ and injective for $i=2$. Let $A$ be an Artin local k-algebra. Then the induced functor $\varphi_{*}: C(L ; A) \rightarrow C(\bar{L} ; A)$ is an equivalence of groupoids depending naturally on $A$.

If $(L, \varepsilon)$ is a $g$-augmented differential graded Lie algebra with zero differential, then Obj $C\left(L^{\prime} ; A\right)$ equals the set of $A$-points over the origin of the quadratic cone $\mathcal{Q}_{L} \subset L^{1}$ defined by $[u, u]=0$. By applying the above theorem with the operation

$$
C \mapsto C \bowtie \exp (\mathfrak{g} \otimes \mathfrak{m})
$$

repeatedly we obtain the following general result, relating quadratic singularities to augmented differential graded Lie algebras:

Proposition. Suppose $(L, d, \varepsilon)$ is a formal g-augmented differential graded Lie algebra. Suppose that $\varepsilon: L^{0} \rightarrow \mathfrak{g}$ is surjective and its restriction to $H^{0}(L) \subset L^{0}$ is injective. Let $\mathcal{Q}$ denote the quadratic cone

$$
\mathcal{Q}_{H(L)} \times \mathfrak{g} / \varepsilon\left(H^{0}(L)\right) .
$$

Then the functors $\mathbf{A}_{\mathbf{k}}^{\text {local }} \rightarrow$ Sets defined by

$$
A \mapsto \mathcal{Q}(A)
$$


and

$$
A \mapsto \operatorname{Iso} C\left(L^{\prime} ; A\right)
$$

are naturally isomorphic.

These general results on differential graded Lie algebras are applied as follows. Let ad $P=P \times_{G} \mathfrak{g}$ be the Lie algebra bundle associated to $P$ by the adjoint representation and $\Omega^{*}(M$; ad $P)$ the differential graded Lie algebra of ad $P$-valued exterior differential forms on $M$ with the covariant differential $D$ associated to $\omega_{0}$. If $\omega \in \mathbf{O b j} \Im_{A}\left(\omega_{0}\right)$, then

$$
\eta=\omega-\tilde{\omega}_{0} \in \Omega^{1}\left(M ; \operatorname{ad} P_{A}\right) \cong \Omega^{1}(M ; \operatorname{ad} P) \otimes A
$$

satisfies (1) and the action of morphisms in $\mathcal{F}_{A}\left(\omega_{0}\right)$ is given by (2). Thus we obtain:

LEMMA. Let $L$ denote the $\mathfrak{g}$-augmented differential graded Lie algebra $\left(\Omega^{*}(M ;\right.$ ad $\left.P), \varepsilon_{p}\right)$. The functor

$$
\mathcal{F}_{A}^{\prime}\left(\omega_{0}\right) \rightarrow C\left(L^{\prime} ; A\right)
$$

defined by $\omega \mapsto \omega-\tilde{\omega}_{0}$ is an isomorphism of groupoids depending naturally on $A$.

The proofs of Theorems 1, 2 and 3 are completed by applying the above propositions to the following.

Proposition. Let $M$ be a compact Kähler manifold and $\rho \in \operatorname{Hom}(\Gamma, G)$ be a representation satisfying the hypotheses of Theorems 1,2 , or 3 . Let ad $P_{\mathbf{C}}$ denote the corresponding flat $\mathfrak{g} \otimes \mathbf{C}$-bundle over $M$ and let $L=\Omega^{*}\left(M ; \operatorname{ad} P_{\mathbf{C}}\right)$. Then the $g_{\mathbf{C}}$-augmented differential graded Lie algebra $\left(L, D, \varepsilon_{p}\right)$ is formal.

The proof follows the ideas indicated in [4] proving that the de Rham algebra of a compact Kähler manifold is formal. In each case the covariant differential on $\Omega^{*}\left(M ;\right.$ ad $\left.P_{\mathbf{C}}\right)$ decomposes as $D=D^{\prime}+D^{\prime \prime}$, where the standard Kähler identities

$$
\Delta_{D}=2 \Delta_{D^{\prime}}=2 \Delta_{D^{\prime \prime}}, \quad\left[\Lambda, D^{\prime}\right]=i D^{\prime \prime *}, \quad\left[\Lambda, D^{\prime \prime}\right]=-i D^{\prime *}
$$

hold. (For Theorems 2 and 3, one uses Deligne's decomposition of $D$ by total bidegree - see Zucker [14], also Corlette [2], Simpson [13].) Since the spaces of $D$-harmonic, $D^{\prime}$-harmonic, and $D^{\prime \prime}$-harmonic forms coincide and the "principle of two types" is satisfied, it follows that

$$
\left(\Omega^{*}\left(M ; \operatorname{ad} P_{\mathbf{C}}\right), D, \varepsilon_{p}\right) \leftarrow\left(\operatorname{Ker} D^{\prime}, D^{\prime \prime}, \varepsilon_{p}\right) \rightarrow\left(H^{*}\left(M ; \operatorname{ad} P_{\mathbf{C}}\right), 0, \varepsilon_{p}\right)
$$

are homomorphisms of $\mathfrak{g}$-augmented differential graded Lie algebras which induce isomorphisms on cohomology.

REMARK. In their paper Symmetry and bifurcation of momentum mappings (Comm. Math. Phys. 78 (1981), 455-478), Arms, Marsden and Moncrief prove quadratic singularities for level sets of momentum mappings for certain Poisson actions on symplectic manifolds. Their results may be understood from the above point of view as follows. To an affine Poisson action 
there is associated an augmented differential graded Lie algebra; their hypothesis (the existence of an invariant positive almost complex structure) implies this differential graded Lie algebra is formal. Details will appear elsewhere.

ACKNOWLEDGMENTS. We have benefited greatly from conversations with many mathematicians. This paper owes its existence to a letter written to us by $\mathrm{P}$. Deligne in which he stated the equivalence theorem above, sketched its proof, indicated how it leads to Theorem 1, and suggested several generalizations. In addition, discussions with M. Artin, E. Bierstone, K. Corlette, D. Gieseker, S. Halperin, S. Kleiman, A. Magid, J. Morgan, M. Nori, H. Rossi, C. Simpson and D. Toledo have been extremely helpful in understanding these ideas.

\section{REFERENCES}

1. M. Artin, On solutions to analytic equations, Invent. Math. 5 (1968), 277-291.

2. K. Corlette, Gauge theory and representations of Kähler groups, The Geometry of Group Representations (Proceedings of Amer. Math. Soc. Summer Conference 1987, Boulder, Colorado), Contemp. Math. (to appear).

3. P. Deligne, Letter to J. Millson and W. Goldman, April 24, 1986.

4. P. Deligne, P. A. Griffiths, J. W. Morgan and D. Sullivan, Rational homotopy type of compact Kähler manifolds, Invent. Math. 29 (1975), 245-274.

5. W. M. Goldman, Representations of fundamental groups of surfaces, Geometry and Topology, Proceedings, University of Maryland 1983-1984 (J. Alexander and J. Harer, eds.), Lecture Notes in Math., vol. 1167, Springer-Verlag, Berlin/Heidelberg/New York, 1985, pp. 95-117.

6. W. M. Goldman and J. J. Millson, Local rigidity of discrete groups acting on complex hyperbolic space, Invent. Math. 88 (1987), 495-520.

7. , Deformations of flat bundles over Kähler manifolds, Geometry and Topology, Manifolds, Varieties and Knots (C. McCrory and T. Shifrin, eds.), Lecture Notes in Pure and Applied Math., vol. 105, Marcel Dekker, New York/Basel, 1987, pp. 129-145.

8. P. A. Griffiths et al., Topics in transcendental algebraic geometry, Ann. of Math. Studies 106 (1984), Princeton Univ. Press, Princeton, New Jersey.

9. D. Johnson and J. Millson, Deformation spaces associated to compact hyperbolic manifolds, Discrete Groups in Geometry and Analysis, Papers in Honor of G. D. Mostow on His Sixtieth Birthday (R. Howe, ed.), Progress in Math., vol. 67, Birkhäuser, Boston/Basel/ Stuttgart, 1987, pp. 48-106.

10. A. Lubotzky and A. Magid, Varieties of representations of finitely generated groups, Mem. Amer. Math. Soc. No. 336 (1985).

11. M. Schlessinger, Functors of Artin rings, Trans. Amer. Math. Soc. 130 (1968), 208222.

12. M. Schlessinger and J. Stasheff, Deformation theory and rational homotopy type (preprint).

13. C. T. Simpson, Systems of Hodge bundles and uniformization, doctoral dissertation, Harvard University, 1987.

14. S. Zucker, Hodge theory with degenerating coefficients, Ann. of .Math. (2) 109 (1979), 415-476.

\footnotetext{
DEPARTMENT OF MATHEMATICS, UNIVERSity OF MARYLAND, COLlege PARK, MARYLAND 20742
}

Permanent address (John J. Millson): DePARTMENT OF MATHEMATICS, UNIVERSITY of California, Los ANgeles, California 90024 